\title{
In utero and perinatal hypoxic brain damage
}

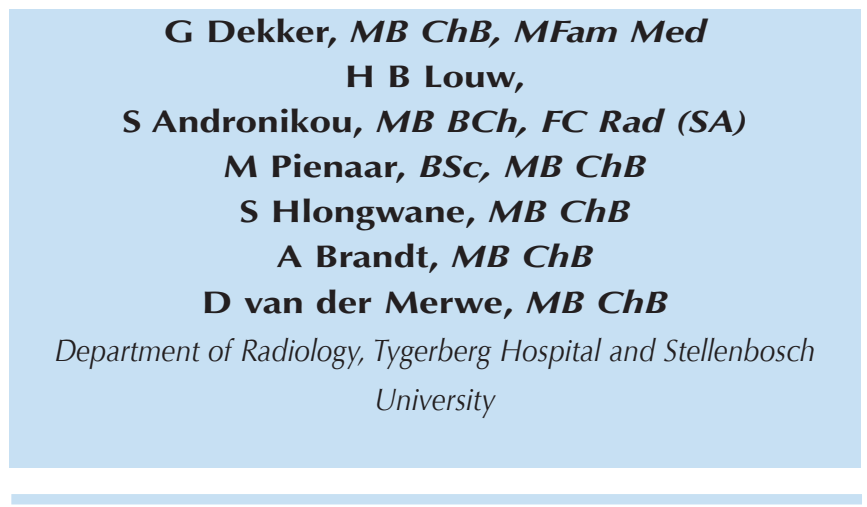

\section{Abstract}

Brain damage due to hypoxia in utero or perinatally often results in specific radiological imaging patterns. The imaging findings are affected by the severity of the insult, the gestational age of the fetus or age of the baby and the time interval between the insult and performing the imaging. This review is aimed at categorising the different patterns of hypoxic brain damage according to the age of the patient at the time of the insult and the severity of the insult.

\section{Introduction}

Brain damage due to hypoxia in utero or perinatally often results in specific radiological imaging patterns. The imaging findings are affected by the severity of the insult, the gestational age of the fetus or age of the baby and the time interval between the insult and performing the imaging.

Most of these patterns are well described in the literature. New advances in imaging modalities have added to the radiologist's diagnostic armature, making it possible to diagnose hypoxic ischaemic damage earlier. This review is aimed at categorising the different patterns of hypoxic brain damage according to the age of the patient at the time of the insult and the severity of the insult. Older as well as newer imaging modalities are included to demonstrate the pathological patterns.

The common radiological appearances of in utero and perinatal cerebral hypoxic/ischaemic changes will be discussed under the following headings:

1. In utero

2. Postnatal

\author{
2.1 Premature (mild hypoxia) \\ 2.2 Premature (severe hypoxia) \\ 2.3 Term (mild hypoxia) \\ 2.4 Term (severe hypoxia)
}

\section{In utero}

Imaging is usually performed post partum, often long after the intra-uterine insult took place.

The results of intra-uterine hypoxic insults before 32 weeks' gestational age are mainly:

- schizencephaly (before 26 weeks)

- porencephaly

- hydranencephaly.

Insults taking place in utero after 32 weeks (much like in the premature neonate) usually lead to:

- multi-cystic encephalomalacia.

A possible explanation for the gestation-dependent difference is the gestational age-related astrocyte response. In a very young fetus necrotic brain tissue is completely resorbed, a process called liquefaction necrosis. There is very limited astrocytic reaction in the brain at this age. This then results in the smooth-walled, fluid-filled cavities of schizencephaly, porencephaly and hydranencephaly. In the more mature brain, characterised by stronger astrocyte proliferation, the post-necrotic cavity contains septations and an irregular wall composed of mainly astrocytes. This may range from pure cysts to cysts with astroglial septae to gliosis without cysts.

The differential diagnosis for these cystic cerebral changes includes: vascular insults, congenital/genetic conditions, infective conditions, e.g. toxoplasmosis and cytomegalovirus (CMV), and toxin exposure..$^{1,2}$

\section{Schizencephaly (Fig. 1a)}

Here the damage usually occurs before 26 weeks' gestational age. This allows for a dysplastic grey matter lining to form on the rim of the cystic cavity. In $60 \%$ of cases it is unilateral and in $40 \%$ bilateral. ${ }^{1,3}$

\section{Porencephaly (Fig. 1b)}

Here the damage usually occurs between 26 and 32 weeks. The resulting appearance is a smooth-walled focal cavity, not lined by grey matter, with minimal surrounding glial reaction. 


\section{PICTORIAL REVIEW}

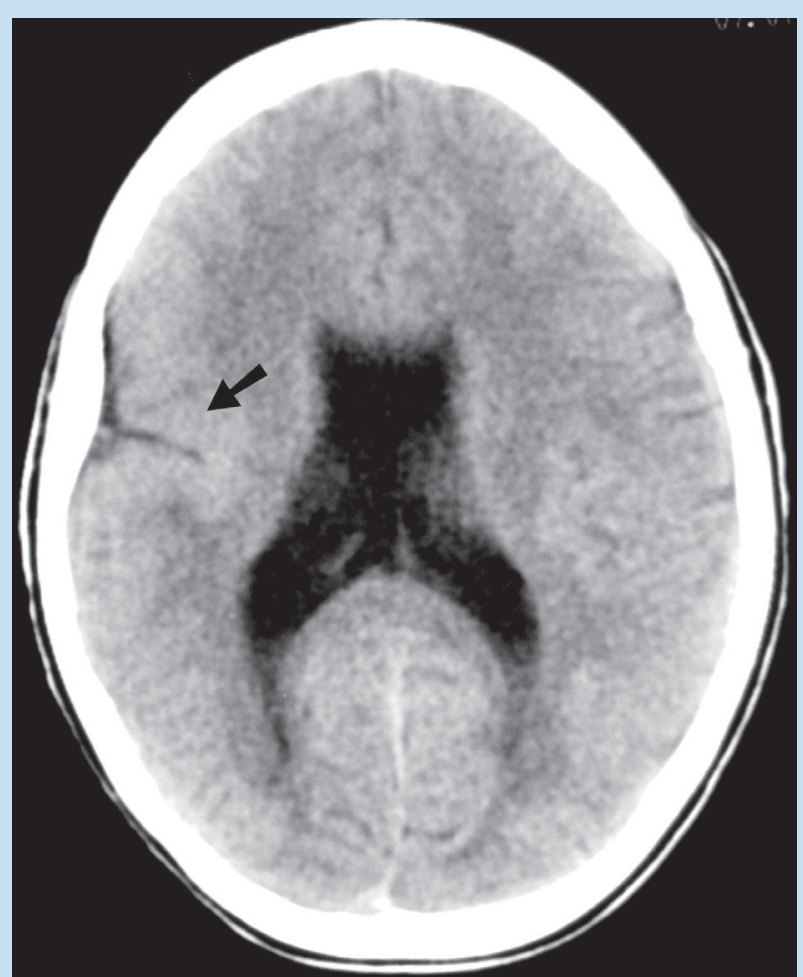

Fig. 1a. Schizenecephalic cleft lined by grey matter is seen on the right (arrow).

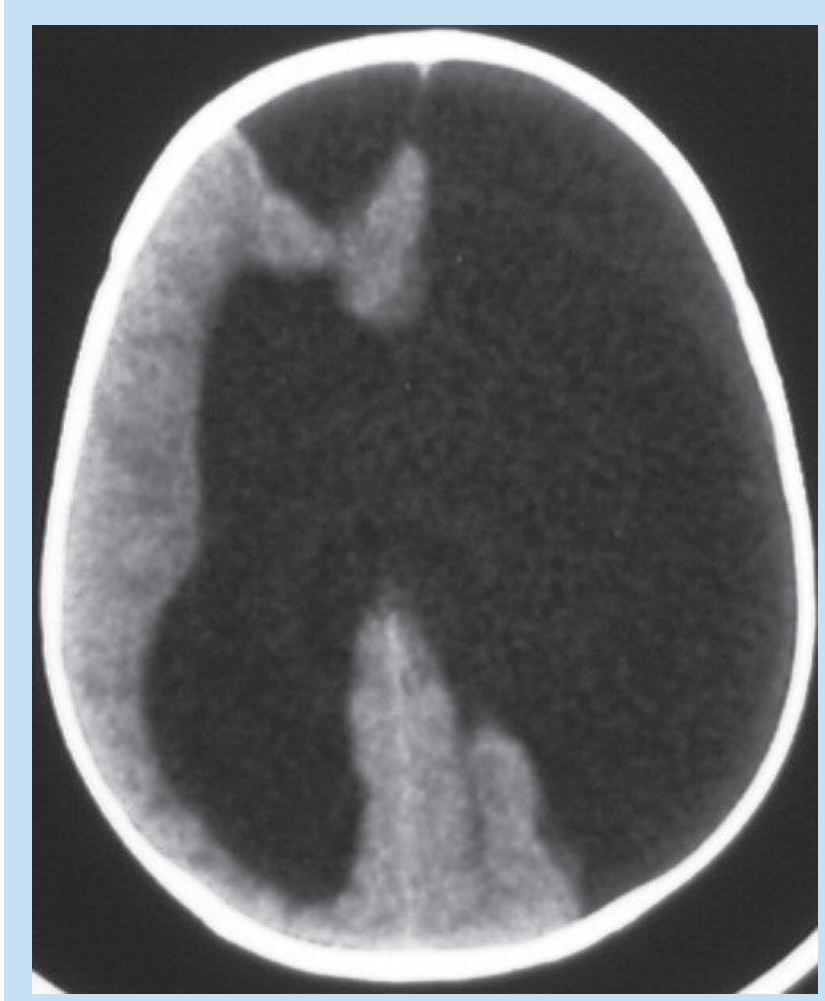

Fig. 1b. Porencephaly: a large CSF collection has replaced the portion of the brain lost due to an in utero insult. The edges are not lined by grey matter.

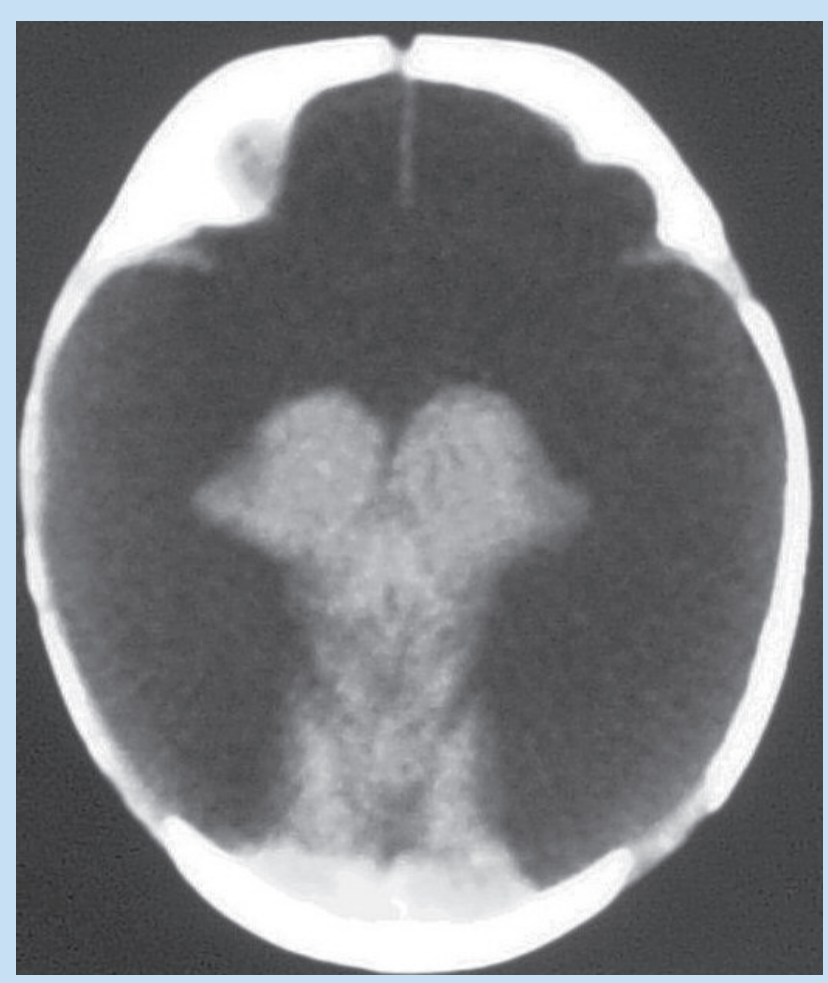

Fig. 1c. Hydranencephaly: there is very little cortical tissue that remains other than the central nuclei and posterior fossa structures after a severe in utero insult.

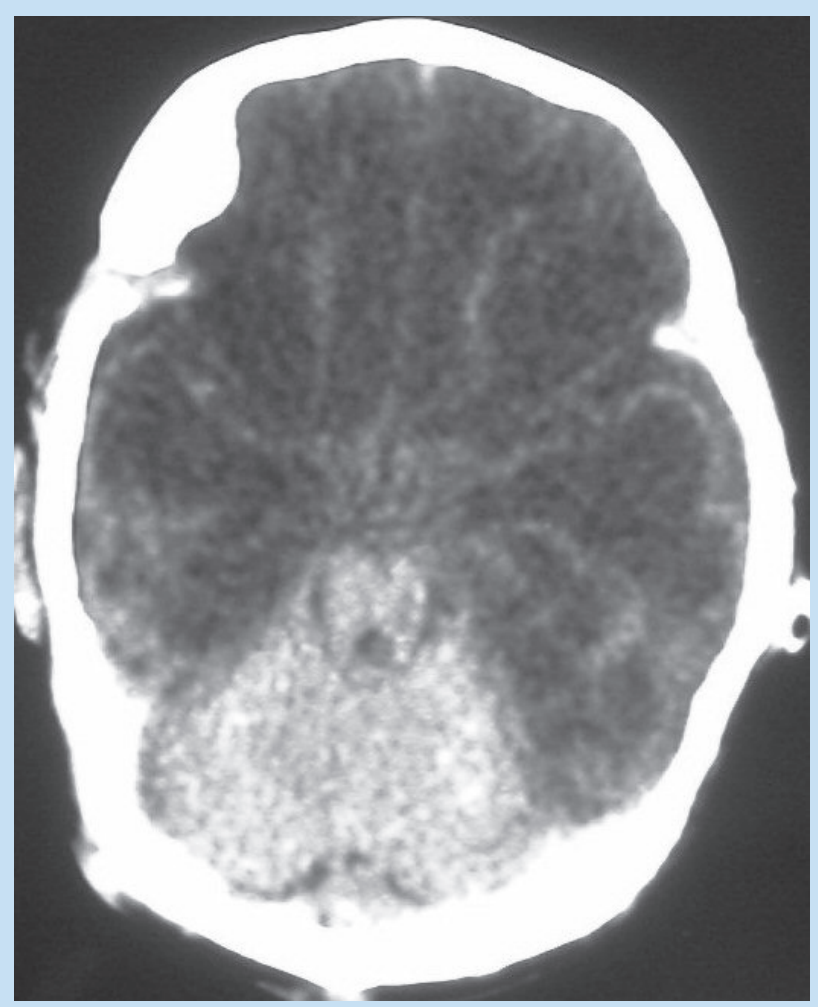

Fig. 1d. Multicystic encephalomalacia: a thin cortical mantle outlines the multicystic areas of predominantly white matter destruction. The ventricular multicystic areas of predominant
outlines are indistinguishable. 


\section{PICTORIAL REVIEW}

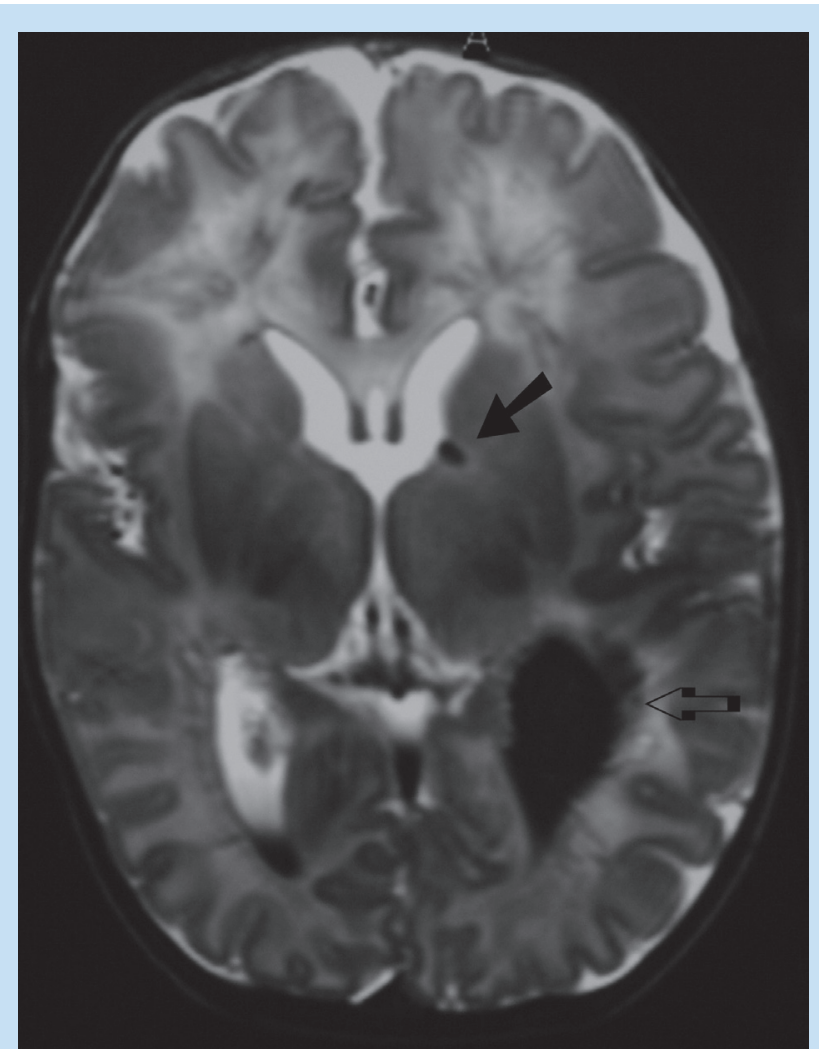

Fig. 2.1a. MRI changes in a preterm neonate: T2 periventricular white matter high signal, subependymal haemorrhage (arrow), left parenchymal haemorrhage and intraventricular haemorrhage (open arrow) in posterior horns of lateral ventricles.

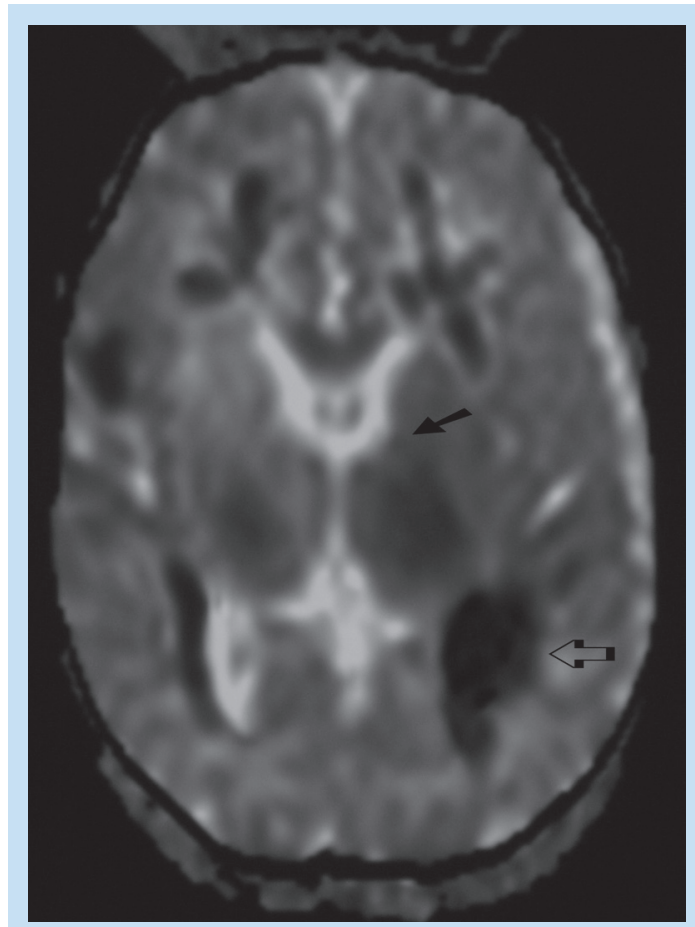

Fig. 2.1b. ADC map shows reversal of DWI signal in periventricular white matter indicating restricted diffusion (hypoxia and cytotoxic oedema). The blackout.

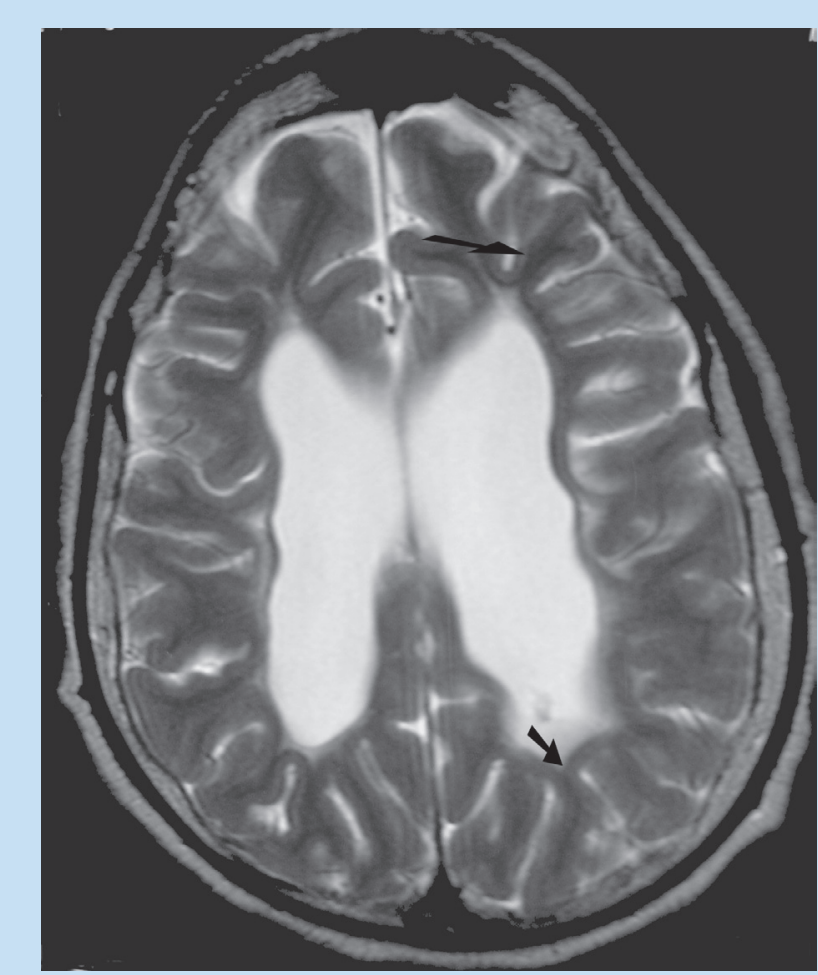

Fig. 2.1c. T2 demonstrating deep sulcus sign (short arrow) as a result of white matter volume loss. Ulegyria (long arrow) [mushroom-shaped gyri] is the consequence of hypoxia at the watershed zones.

\section{Hydranencephaly (Fig. 1c)}

This is often called 'porencephaly of nearly the entire cerebrum.'. It is characterised by absence of brain supplied by anterior/middle cerebral artery. Brain normally supplied by the posterior circulation is often intact: posterior temporal lobes, occipital lobes, thalami, falx cerebri and infratentorial structures. Thin-walled sacs containing CSF largely replace the cerebral hemispheres. The cortical mantle and ventricles are absent. ${ }^{4}$

It is important to distinguish hydranencephaly from severe hydrocephalus. The latter has a thin rim of cortex sometimes only seen on magnetic resonance imaging (MRI). ${ }^{1}$

\section{Multicystic encephalomalacia (Fig. 1d)}

This is characterised by astrocyte proliferation and glial septations in/around damaged tissue leading to cyst formation termed multicystic encephalomalacia.

\section{Postnatal}

\section{Susceptible areas}

Certain areas in the brain are more susceptible to ischaemic/hypoxic events depending on the age of the 


\section{PICTORIAL REVIEW}

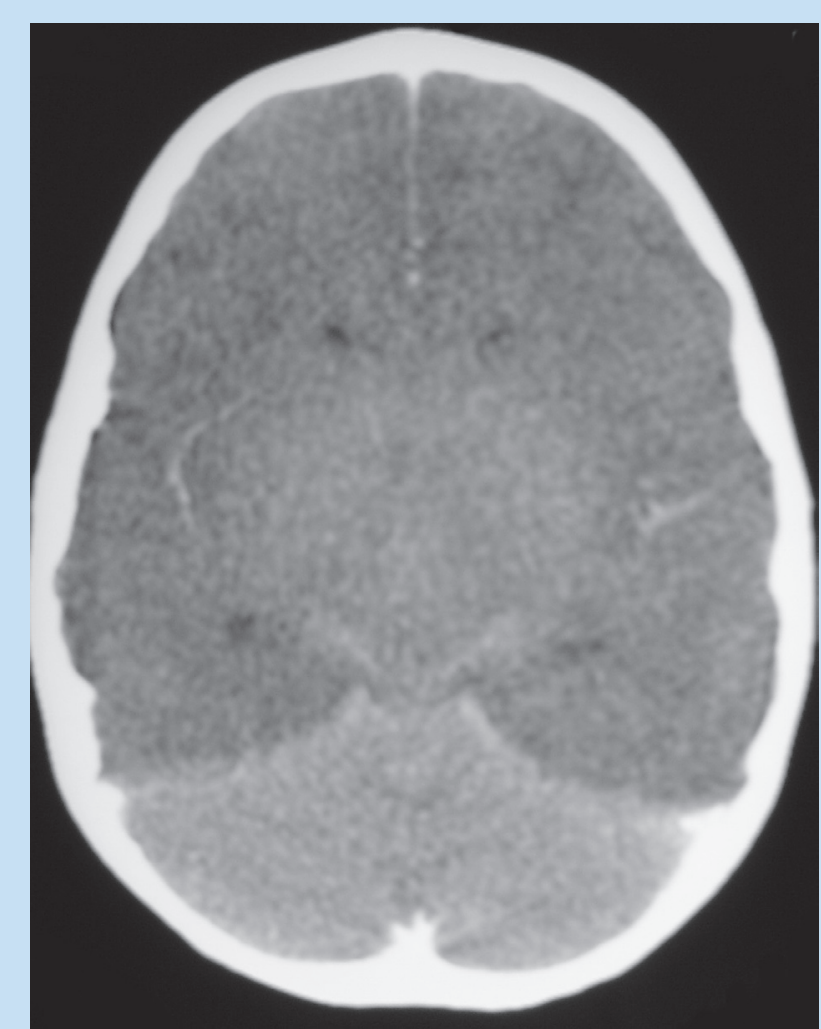

Fig. 2.2a. The bright cerebellum sign indicates normal appearance of the posterior fossa structures with severe oedema of the supratentorial brain resulting in low density, loss of grey/white interface and obliteration of surface markings and sulci.

patient. ${ }^{1-4}$ In the premature neonate changes are mainly seen in the periventricular white matter where, as in term neonates, the watershed zones between major vascular territories are affected. It is important to remember that in preterm neonates the watershed zones are still in the central part of the brain, hence the white matter damage. In all age groups metabolically active areas like the basal ganglia and perirolandic cortex may be affected, especially in the case of severe hypoxia. Although all cases of hypoxia leading to brain damage are to be regarded as clinically severe, most authors of paediatric neuroradiology textbooks make a distinction between $\mathrm{mild} /$ moderate and severe/profound hypoxia.

\subsection{Preterm (mild/moderate hypoxia)}

There is some overlap in the appearance between damage in this period and damage after 32 weeks in utero. It is characterised by astrocyte proliferation and glial septations in and around damaged tissue, leading to cyst formation termed multicystic encephalomalacia.

Periventricular leucomalacia (PVL) is the term used for periventricular white matter necrosis that occurs

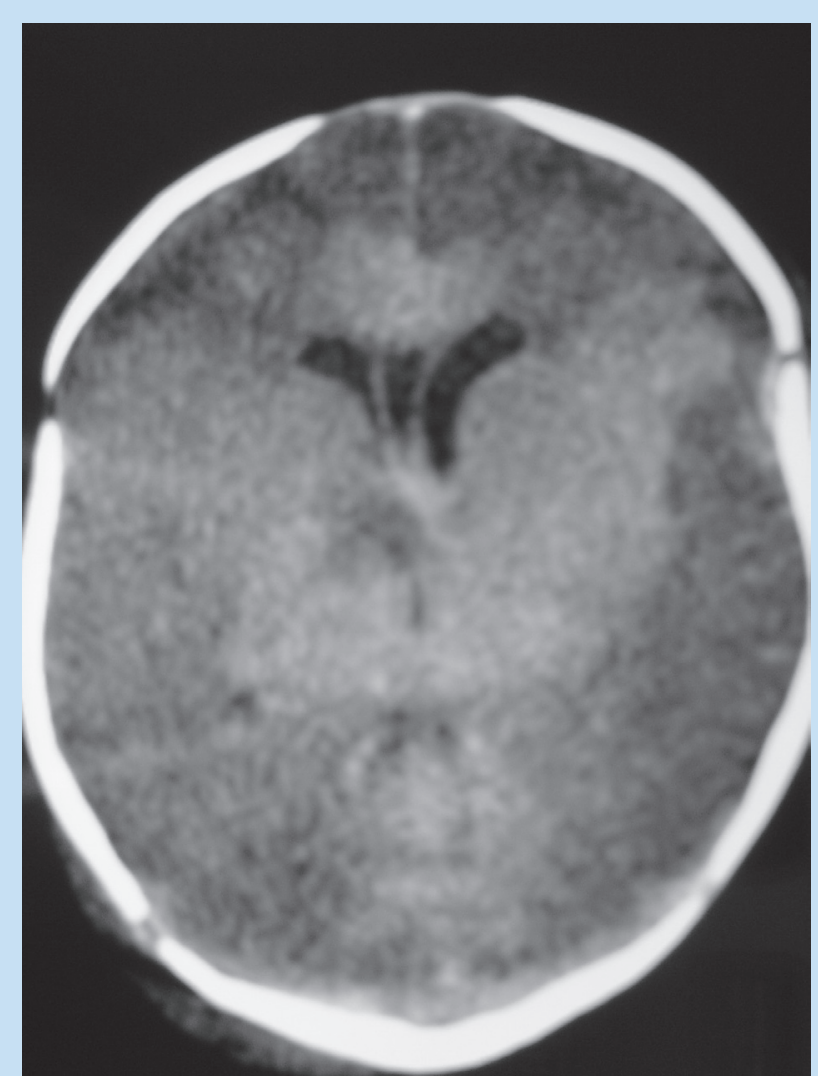

Fig. 2.2b. In the reversal sign the central structures including the grey matter nuclei and white matter are dense relative to the peripheral cortex which is of low density (usually cortex has a higher CT density than white matter).

after hypoxic events in very-low-birth-weight premature babies. $^{5}$ PVL occurs mainly around the trigone of the lateral ventricles, in other words, posteriorly. ${ }^{2,3,6}$ PVL may also occur adjacent to the frontal horns and may even be diffusely distributed. ${ }^{5}$ PVL is usually due to watershed ischaemia in the perforating arteries. ${ }^{2}$

\section{Ultrasound}

Ultrasound is better in the acute setting than computed tomography (CT) or MRI. (The latter is better in late stages. $\left.{ }^{1,3}\right)$ During the first 2 days after injury ultrasound shows an increase in echogenicity (periventricular flare ${ }^{5}$ ), periventricular white matter being brighter than choroid plexus. ${ }^{1-4}$

After a few days cystic cavitation takes place. ${ }^{2,5}$ This is the most specific ultrasound sign. ${ }^{5}$ Ventriculomegaly is a very sensitive sign as the ventricles expand towards the areas of volume loss over time. ${ }^{3}$

The following ultrasound classification for PVL may be used for prognostic purposes:1,2 


\section{PICTORIAL REVIEW}

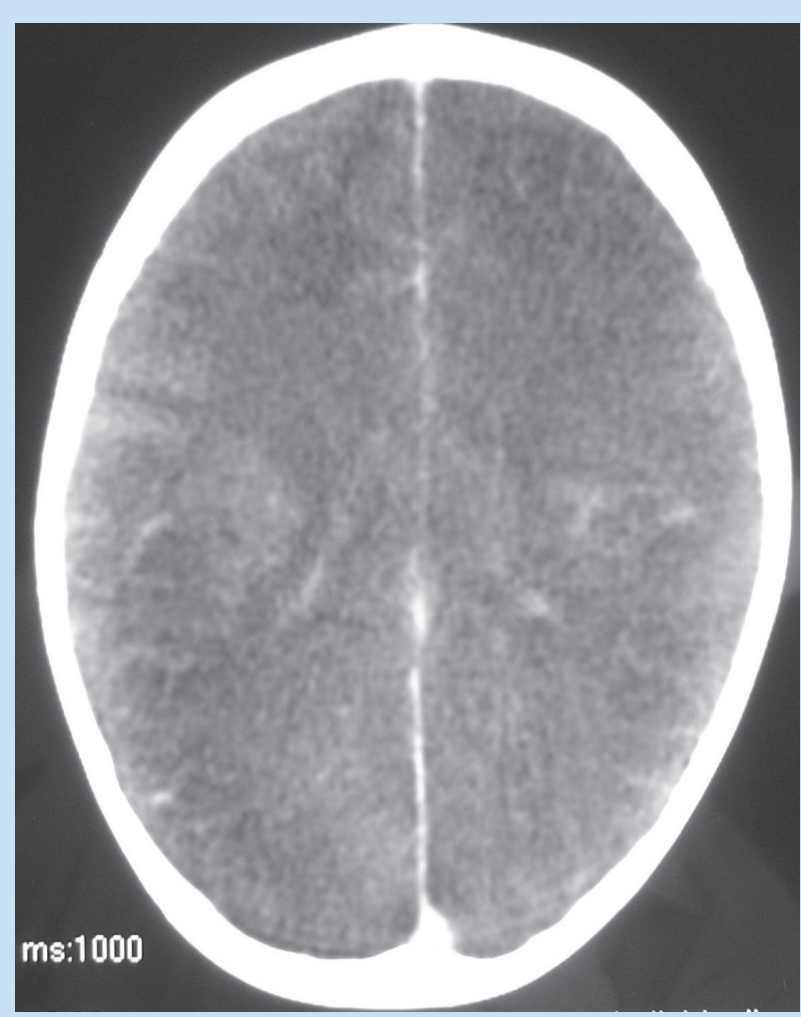

Fig. 2.3a. Axial postcontrast CT demonstrates the loss of the cortical ribbon density at both the anterior and posterior watershed areas bilaterally, accompanied by localised swelling.

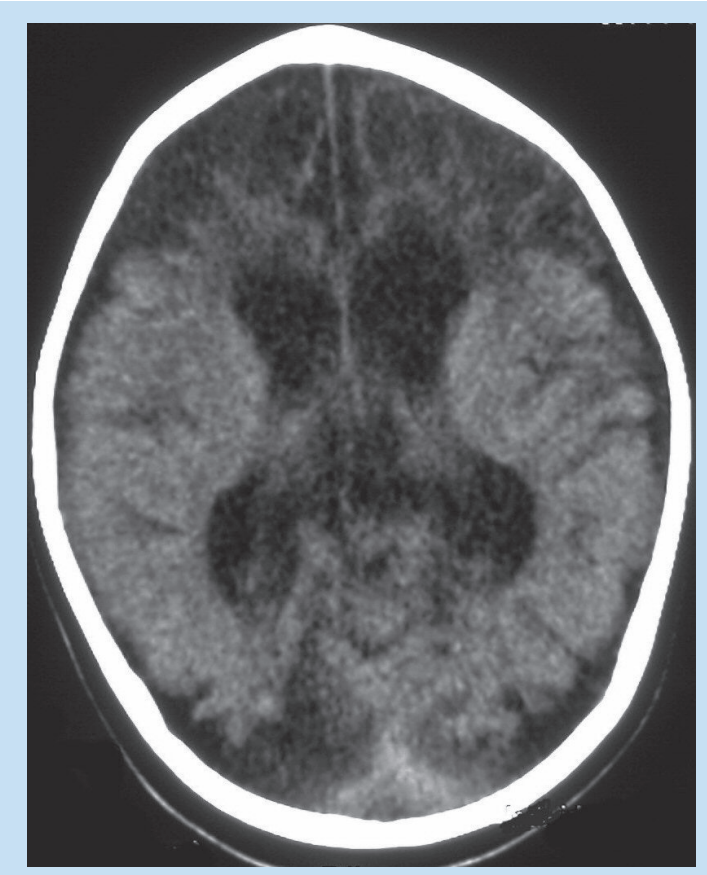

Fig. 2.3b. Delayed non-contrast CT demonstrated cystic changes bilaterally in the anterior watershed areas and pronounced localised atrophy in the posterior watershed areas. The ventricles are large either from ex vacuo dilatation or as a result of hydrocephalus caused by intraventricular haemorrhage.

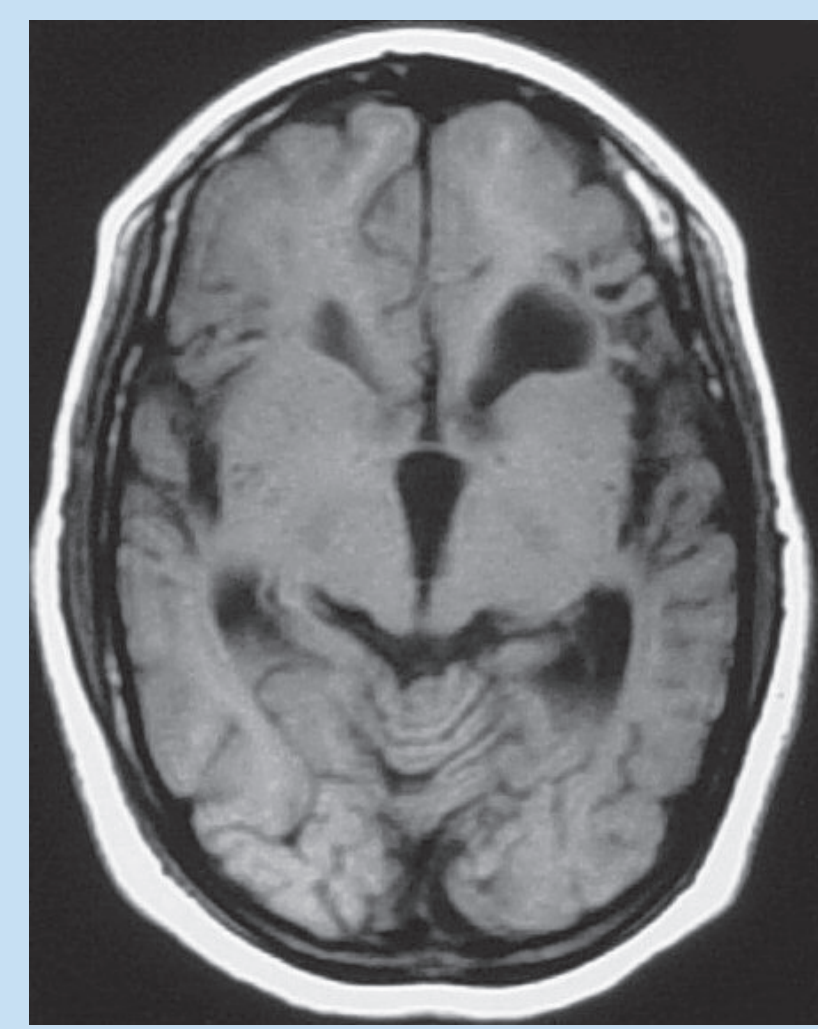

Fig. 2.3c. Ulegyria (mushroom-shaped gyri) are seen at the anterior watershed areas. The deep portion of the gyrus is more affected than the superficial portion due to different susceptibility to hypoxia.

GR 1: Periventricular increased echogenicity lasting less than 7 days

GR 2: Periventricular increased echogenicity lasting more than 7 days

GR 3: Small periventricular cysts

GR 4: Extensive periventricular cysts

GR 5: Periventricular lesions involving subcortical white matter - extensive periventricular and subcortical cysts.

\section{CT}

Early CT is of limited value. ${ }^{5}$ Later it may demonstrate periventricular white matter volume loss, lateral ventricle dilatation ${ }^{2,5}$ and cyst formation. ${ }^{1,3}$

\section{MRI}

Early changes. Restricted diffusion on diffusion weighted imaging (affected areas appear hyperintense), confirmed by ADC (apparent diffusion coefficient) map (affected areas appear hypo-intense), is the best early MRI sign. ${ }^{5}$ Increased signal occurs of the periventricular white matter on T1W in the event of haemor- 


\section{PICTORIAL REVIEW}

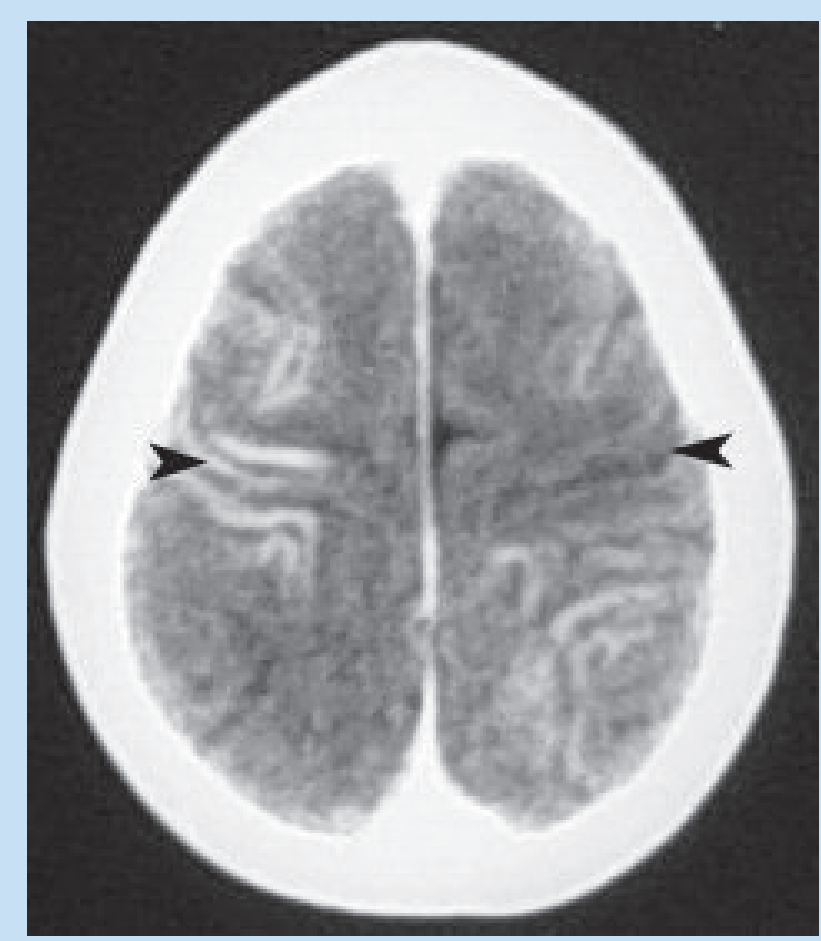

Fig. 2.4a. There is abnormal enhancement at the perirolandic region bilaterally due to susceptibility to hypoxia because of the increased metabolic needs.

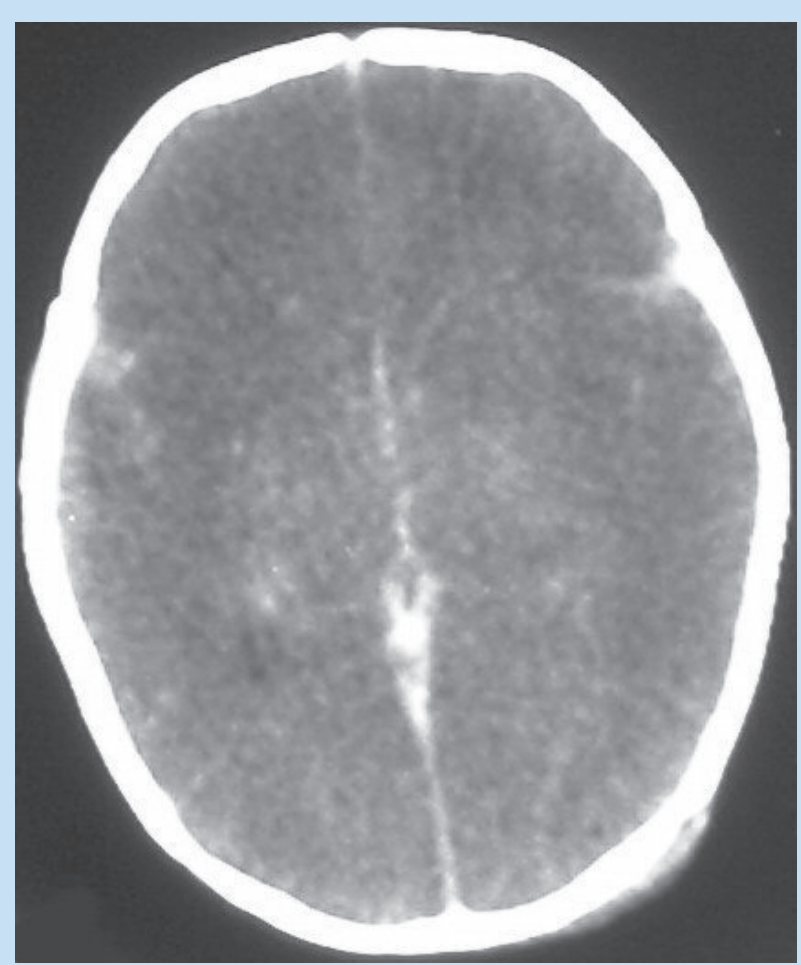

Fig. 2.4b. Complete loss of cortical density and effacement of the surface markings represent severe oedema as result of hypoxia.

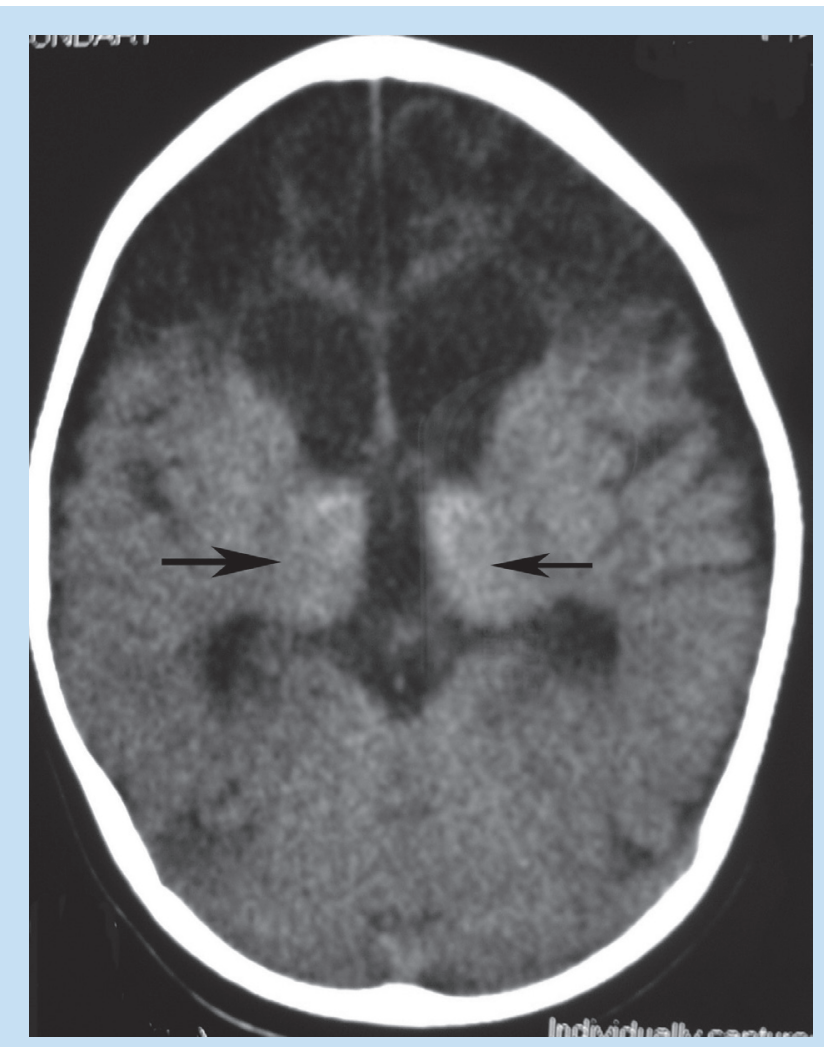

Fig. 2.4c. In addition to the anterior watershed subcortical cystic change and volume loss, there is increased density of the thalami (arrows) representing mineral deposition (calcification) in this area, known for its susceptibility to hypoxia.

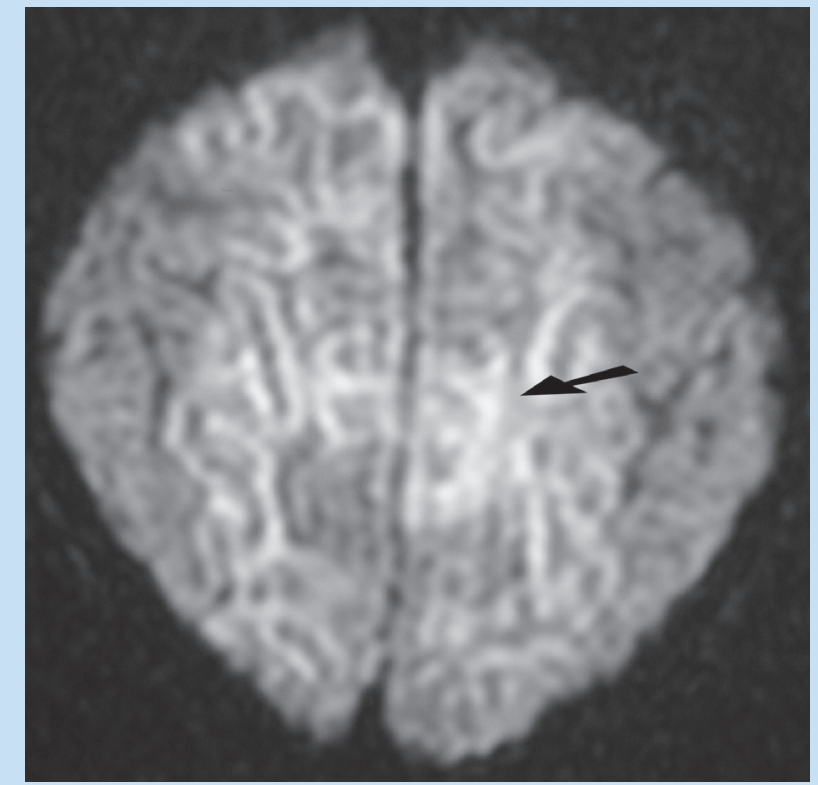

Fig. 2.4d. DWI demonstrated signal abnormality in the perirolandic region (arrow) which is susceptible to hypoxic injury. 


\section{PICTORIAL REVIEW}

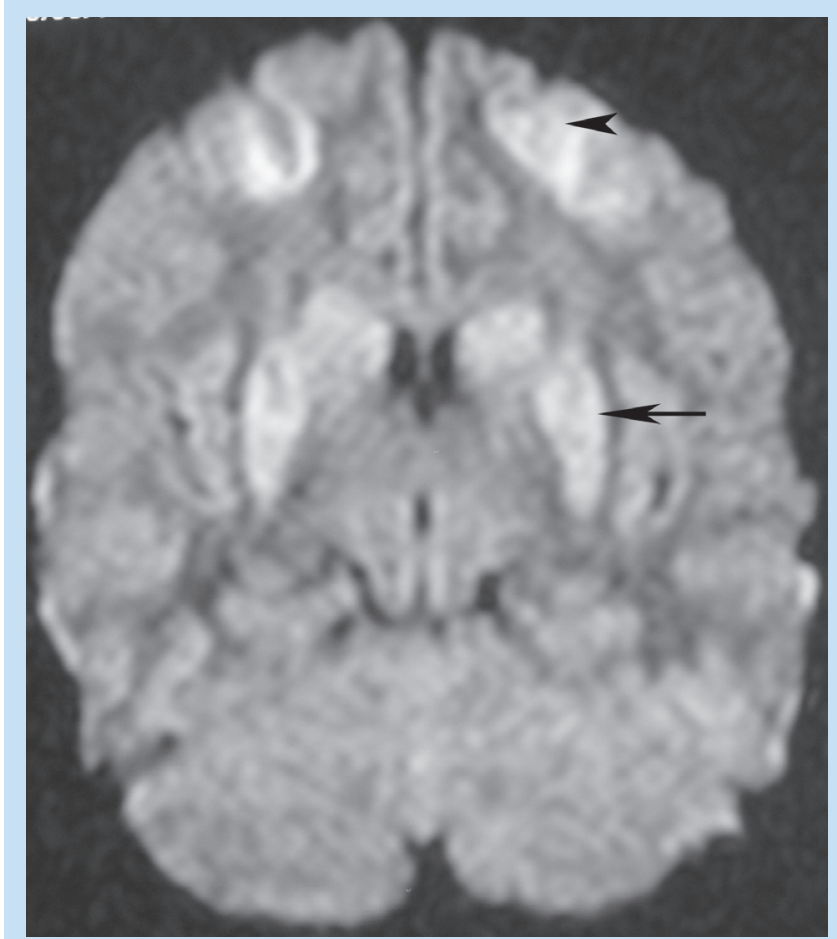

Fig. 2.4e. DWI demonstrates abnormality at the anterior watershed region (arrowhead) bilaterally and in the basal ganglia (arrow) bilaterally due to a global severe hypoxic event.

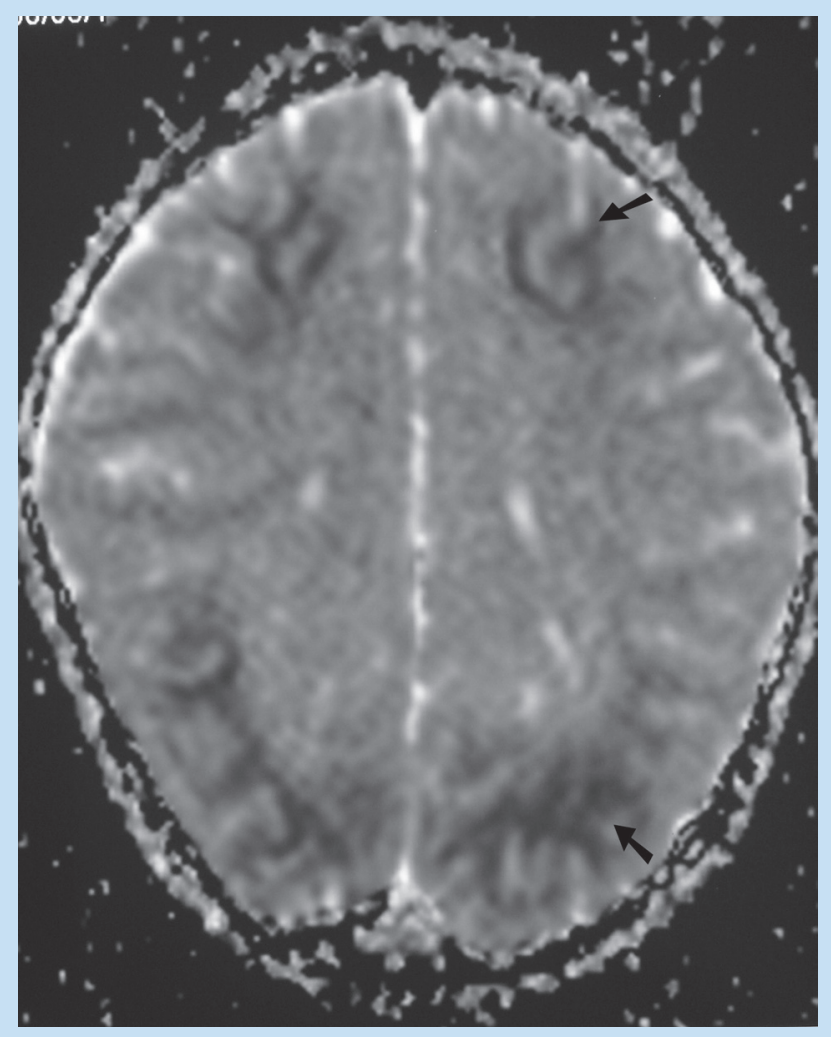

Fig. 2.4f. ADC map demonstrates inversion of the high signal abnormality seen at both anterior and posterior watershed regions (arrows) on DWI in a patient with a global hypoxic event. Reversal of the high DWI signal to a low ADC map signal confirms restricted diffusion associated with ischaemic damage.

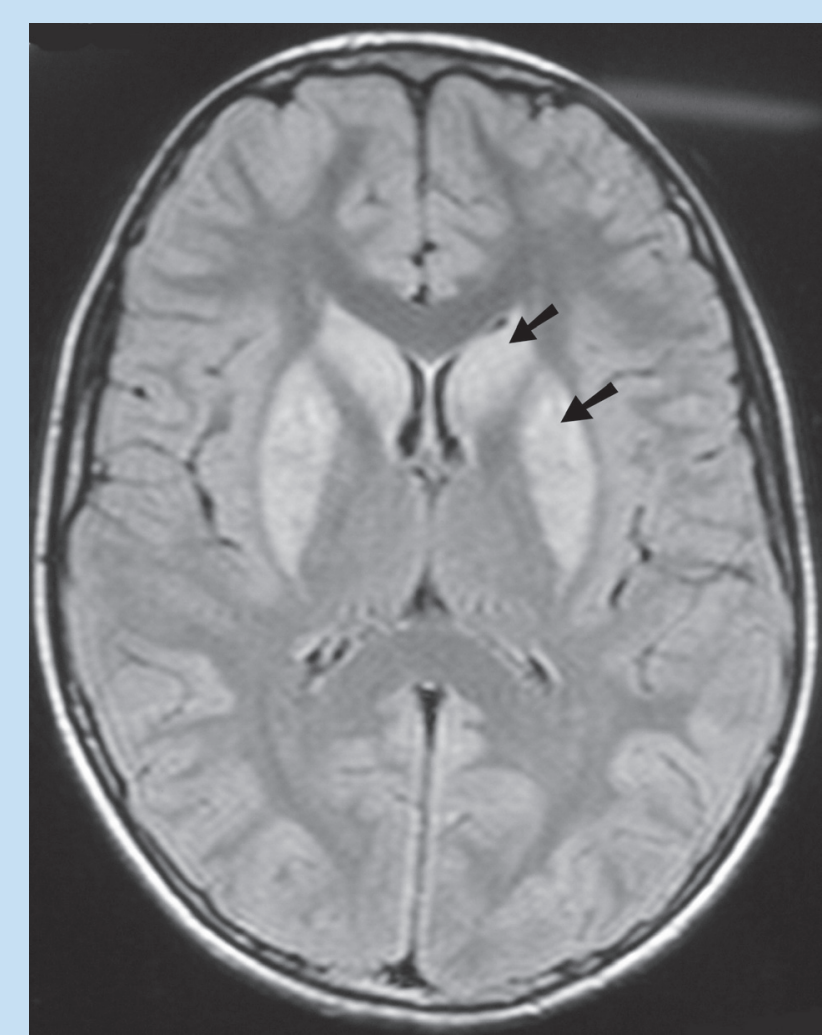

Fig. 2.4g. Fluid-attenuated inversion recovery (FLAIR) demonstrates bilateral symmetrical involvement of the basal ganglia associated with severe hypoxia.

rhagic necrosis ${ }^{5}$ which may appear as punctate areas of increased signal on T2W after 7 days $^{1}$ (Figs 2.1a and 2.1b).

Late changes. Periventricular white matter volume loss with or without cavitation, thin corpus callosum and ventriculomegaly. ${ }^{1.5}$ Cyst regression leads to white matter volume loss ${ }^{1,3}$ seen best on T2W. ${ }^{3}$ The deep sulci, abutting the ventricles, are useful to determine white matter volume loss $^{3}$ (Fig. 2.1c).

\subsection{Preterm (severe hypoxia)}

Although some authors do not distinguish between mild and severe hypoxia, ${ }^{5}$ profound hypoxia before 32 weeks' gestational age may lead to imaging changes, often bilateral, ${ }^{2}$ of the thalami, basal ganglia and posterior brainstem..$^{1.3}$ as well as subcortical white matter. ${ }^{2}$

\section{Ultrasound}

Increased echogenicity of the affected areas is seen early. ${ }^{1,2}$ 


\section{CT}

Loss of grey/white matter interface secondary to oedema and haemorrhage is a common early finding. ${ }^{3}$ Periventricular white matter appears hypodense ${ }^{3}$ and hypodense basal ganglia compare isodense with white matter. ${ }^{1-3}$ Posterior fossa structures may appear white when preferentially spared. This is called the white cerebellar sign (Fig. 2.2a).

The reversal sign may be seen in acute cases of severe hypoxia when central venous congestion dominates the CT appearance ${ }^{3}$ and there is peripheral hypodensity and central hyperdensity, the opposite of the normal appearance (Fig. 2.2b).

\section{MRI}

Early changes. At $<2$ days: restricted diffusion (high signal), increased T2 signal of affected areas 1 . At $>2$ days: increased T1 signal.

Late changes. Injured tissue (liquefaction necrosis/ astrogliosis) leads to ventriculomegaly, volume loss of thalami and cerebellum, ${ }^{1}$ gliosis, thalamic scarring and demyelination. ${ }^{5}$

\subsection{Term (mild hypoxia)}

In mild hypoxic injury in term babies (for example as seen with prolonged partum, some showing signs of fetal distress prior to delivery, some requiring resuscitation at birth ${ }^{7}$ ), the most common anatomical distribution of radiological abnormalities is in the watershed distribution of cortex and white matter. ${ }^{2,3,7}$

\section{Ultrasound}

There is increased echogenicity of gyral white matter, cortex and sulci.' It is difficult to detect on ultrasound in term babies due because of affected areas being near to the inner table of the skull and midline. ${ }^{1}$ Ultrasound is more useful in preterm babies.

\section{CT}

Early changes. Low-density changes are seen in the watershed areas (white matter and cortex), with sparing of the thalami and basal gangliaa, (Fig. 2.3a).

Late changes. Atrophic changes occur in the peritrigonal/occipitoparietal as well as frontoparietal areas with intact thalami and basal ganglia. ${ }^{1,3}$ Secondary $e x$ vacuo dilatation of occipital horns may occur ${ }^{1}$ (Fig. 2.3b). Ulegyria ${ }^{2,3,7}$ (mushroom-shaped gyri) may be seen in parasagittal cortex as a result of more pronounced atrophy of the deep compared with the superficial portion of the gyri (Fig. 2.3c).

\section{MRI}

Early changes. DWI/T2W: Affected cortex displays high signal. ADC map distinguishes between T2W shinethrough and ischaemia - if the affected area appears black on ADC map, this suggests true ischaemia. ${ }^{1,3}$ Oedematous brain causes a low signal to normal white matter on T1W. ${ }^{1} \mathrm{~T} 1 \mathrm{~W}$ demonstrates increased signal at the bottom of sulci (laminar necrosis) ${ }^{7}$ leading to ulegyria later. T2W shows blurring of grey/white matter interface.

Late changes. $\mathrm{T} 2 \mathrm{~W}$ is the best sequence to demonstrate border zone gliosis and/or cystic encephalomalacia. $^{7}$

\section{MR spectroscopy}

This investigation is an adjuvant to MRI and gives chemical information in graph form about tissues identified on MRI. Lactate elevation (doublet) is not normally seen and indicates hypoxia/ischaemia. ${ }^{3}$

\subsection{Term (severe hypoxia)}

In addition to watershed damage the following high metabolic rate areas may be affected: lateral thalami ${ }^{1,23}$ corpus striatum, ${ }^{2}$ hippocampus, ${ }^{1,2,7,8}$ dentate nuclei of cerebellum, ${ }^{2}$ deep grey matter, ${ }^{1,7}$ dorsal brainstem, ${ }^{1,3,7}$ perirolandic cortex ${ }^{1,3,7}$ and basal ganglia (Fig. 2.4a).

\section{CT}

Common findings are decreased density of deep grey matter, ${ }^{2,7}$ blurred grey/white matter interface (Fig. 2.4b CT term severe acute, oedema) and occasionally petechial bleeds. The white matter and cortex ${ }^{2,7}$ are usually spared except around the central sulcus region.

The white cerebellum and reversal signs, ${ }^{1-3,7}$ as well as basal ganglia haemorrhage, ${ }^{6}$ may occur in the acute phase and indicate a poor prognosis.

Chronic changes would include volume loss and/or calcification of the basal ganglia, (Fig. 2.4c) thalami and posterior brainstem.

\section{MRI}

Early changes. At $<2$ days: DWI demonstrates restricted diffusion even if T1W/ T2W appear normal. ${ }^{79}$ Oedematous tissue appears bright on DWI. This is then confirmed on ADC map, where it appears dark (Figs 2.4d, 2.4e and 2.4f). Elevated signal is seen on T2W of 


\section{PICTORIAL REVIEW}

the thalami, lentiform nuclei and perirolandic cortex with sometimes sparing of cerebral cortex ${ }^{1,2,3}$ (Fig. 2.4g).

On $\mathrm{T} 2 \mathrm{~W}$ sequence the basal ganglia and thalami may appear iso-intense to white matter instead of hypointense. ${ }^{1}$

Late changes. Persistent elevated signal on T1W and $\mathrm{T} 2 \mathrm{~W}$ and shrinkage of the affected areas.

\section{MR spectroscopy}

MR spectroscopy may be more sensitive than MR in detecting an insult due to HIE. Lactate in full-term $(>37$ weeks) neonates may be the only finding in the first 24 hours. ${ }^{7}$ Lactate may be a normal finding of developing brain $<37$ weeks. ${ }^{710,11}$

\section{Prognostic value}

Developmental abnormalities are observed in the majority of infants with periventricular leucomalacia and focal parenchymal haemorrhages. An even higher incidence of clinical abnormalities is seen with basal ganglia haemorrhage and multicystic encephalomalacia. ${ }^{6}$ Contrast enhancement of basal ganglia or brainstem in cases of severe hypoxia in premature neonates seems to indicate a worse outcome clinically. ${ }^{2}$

\section{Conclusion}

Although in hypoxic ischaemic cerebral damage there are many areas of overlap between the different categories discussed, it is useful for the junior radiologist or registrar to have a simplified, dogmatic understanding of the topic. This classification based on age, severity and timing of the imaging provides such a basic framework. Despite criticism of the mild/severe classification, it is still widely used by the leading paediatric neuroradiology authors.

The type of imaging that best depicts the abnormalities varies tremendously among the different categories. For instance, the use of DWI as well as MR spectroscopy makes it possible to make a diagnosis earlier after the insult than with the conventional $\mathrm{T} 1$ or $\mathrm{T} 2$ sequences. A good classification thus assists in the choice of imaging to be performed.

\section{Acknowledgements}

Professor RH Hewlett, Department of Pathology, Tygerberg Hospital and University of Stellenbosch.

Dr Roy Giles, Mss Petra de Klerk, Marlise Coetzee and Leoni Zaaiman, Drs Schnetler, Corbett and Partners.

\section{REFERENCES}

1. Barkovich AJ. Pediatric Neuroimaging. 3rd ed. Philadelphia. Lippincott Williams and Wilkins, 2000. 157208.

2. Grossman RI, Yousem DM. Neuroradiology: The Requisites. 2nd ed. Philadephia: Elsevier Inc, 2003. 444445

3. Giles R. Hypoxia/ischemia in premature infants, term infants and the older child. In: Andronikou S et al. Baby Steps into Paediatric Neuroradiology. Cape Town: SAMA Health and Medical Publishing Group, 2004: 19-24.

4. Illner A. Hydranencephaly. In: Osborn AG et al. eds. Diagnostic imaging. Brain. Salt Lake City, Utah: AMIRSYS, 2004: I 4: 66-67.

5. Hedlund GL. HIE, Preterm. In: Osborn AG et al., eds. Diagnostic imaging. Brain. Salt Lake City, Utah: AMIRSYS, 2004: I 4: 68-71.

6. Keeney SE, Adcock EW, McArdle CW. Prospective observations of 100 high-risk neonates by high field (1.5 Tesla) magnetic resonance imaging of the central nervous system. II. Lesions associated with hypoxicischemic encephalopathy. Paediatrics 1991; 87 (4): 431-438.

7. Blaser SI. HIE, Term. In: Osborn AG et al. eds., Diagnostic imaging. Brain. Salt Lake City, Utah: AMIRSYS, 2004. I 4: 72-75.

8. Maneru C, Serra-Grabulosa JM, Junque C et al. Residual hippocampal atrophy in asphyxiated term neonates. J Neuroimaging 2003; 13: 68-74.

9. Bozzao A, Di Paolo A, Mazzoleni C et al. Diffusion-weighted MR imaging in the early diagnosis of periventricular leucomalacia. Eur Radiol 2003; 13: 1571-6.

10. Fan G, Wu Z, Chen L et al. Hypoxia- ischemic encephalopathy in full-term neonate: correlation proton MR spectroscopy with MR imaging. Eur J Radiol 2003; 45: 91-8.

11. Malik GK, Pandey M, Kumar R et al. MR imaging and in vivo proton spectroscopy of the brain in neonates with hypoxic ischemic encephalopathy. Eur J Radiol 2002; 43: 6-13. 